\begin{tabular}{ll} 
Changing the culture landscape & 1035 \\
\hline A moving experience & 1037 \\
\hline Making a big production of it & 1040 \\
\hline Productivity at your disposal & 1042 \\
\hline Box 1: Getting organized & 1037 \\
\hline
\end{tabular}

\title{
Thinking outside the dish
}

\author{
Getting cultured cells to 'do their job'-either to recreate in vivo function in an artificial setting or to \\ churn out immense volumes of protein on command-requires both an understanding of the demands \\ of cell physiology and the technology to meet those demands. Michael Eisenstein looks at contemporary \\ solutions for culture conundrums.
}

Your culture dish could be lying to you. Perhaps this is an overly strong statement to make; after all, for most basic research projects, conventional Petri or flask cultures are more than adequate. But for many investigators hoping to study physiological or pathological processes, it is becoming increasingly clear that good science may require more 'three-dimensional' thinking.

Mina Bissell of the Lawrence Berkeley Laboratory recalls being taken aback when, more than thirty years ago, she first became acquainted with how most biologists were culturing their mammalian cells: on flat plastic surfaces utterly devoid of any biological context. Work by colleagues like Beatrice Mintz, who demonstrated a context-dependent component to tumor formation through a series of implantation experiments in mice, inspired Bissell and her colleagues to explore the influence of extracellular environment on cellular phenotype. What they found was striking-multiple lines of evidence indicating that the development and behavior of many cells is modulated by their interactions with the extracellular matrix, a surrounding network of proteins and macromolecules that directly associates with a variety of cellsurface receptors.

According to Bissell, these findings had a profound impact on her breast cancer research. "We can take a malignant cell and make it normal just by changing the surrounding structure, and we can take a normal cell and destroy that structure and make it malignant," she says. "Our current model is that tissue structure and organ structure are really dominant over genotype-which is a paradigm shift, because it tells you that people studying signaling in the wrong context may get the wrong answers."

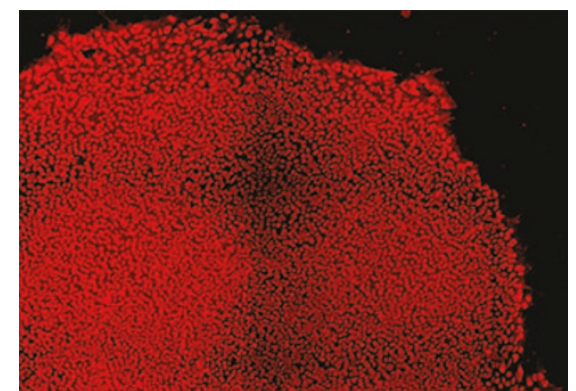

H9 hESCs cultured on BD BioCoat Matrigel Matrix for ES Cell Culture stain positive for 0ct-3/4, a marker of undifferentiated hESCs. (Courtesy of BD Biosciences.)

\section{Changing the culture landscape}

Several companies, such as R\&D Systems, Nunc, BD Biosciences and Corning offer specially treated plates and inserts that have been coated with molecules that improve cellular affinity for glass or plastic, and may partially recapitulate the effects of a physiological matrix on cell growth. These may consist of actual extracellular matrix components, such as collagen and fibronectin, or of synthetic substrates such as positively charged poly(Llysine) or poly(D-lysine) amino acid chains, and are a popular option for researchers working with primary cells.

Hynda Kleinman and George Martin first demonstrated the value of reconstituted basement membranes from EngelbrethHolm-Swarm (EHS) mouse tumor extracts as a culture substrate in the mid-1980s (ref. 1 ), and their use has proven a considerable boon to cancer researchers like Bissell. For example, Bissell's group has shown that normal breast epithelia, which remain nonpolarized and poorly differentiated in two-dimensional culture, will assemble into spherical, polarized cysts called acini that secrete milk proteins when cultured on basement membranes, whereas malignant cells instead form disorganized proliferative masses ${ }^{2}$.

BD Biosciences also offers an EHS tumorderived extract, BD Matrigel Matrix, as a substrate for their BD BioCoat product line. "It has a very rich content of [extracellular matrix] molecules such as laminin, collagen and growth factors," explains technical products and programs manager Marshall Kosovsky. "We have loyal customers who have been using this product for over 15 years to support the growth and differentiation of mammary epithelial cells, hepatocytes, endothelial cells and other cell types." Similar EHS tumor-derived basement membrane extracts are also available from other manufacturers, including Trevigen's Cultrex products or Millipore's ECMatrix.

Matrigel has also proven useful for the culture of human embryonic stem cells (hESCs), and James Thomson and colleagues at the University of Wisconsin at Madison have worked extensively with this substrate for the long-term maintenance of pluripotent hESCs in the absence of mouse fibroblast feeder cells. All the same, Matrigel is not a perfect solution - as a reconstituted extract, its contents are poorly defined, and the use of animal-derived products remains an ongoing issue for many scientists. Thomson and colleagues have subsequently addressed these issues through the reconstitution of extracellular matrix with human tissue-derived matrix proteins ${ }^{3}$, but this remains a partial and impractical solution.

BD Biosciences offers one alternative with their fully synthetic BD PuraMatrix Peptide Hydrogel, which was originally developed 
by 3DM Synthetic Nanoscaffolds, Inc. PuraMatrix is 99\% water, with the remaining percentage consisting of short oligopeptide fragments that self-assemble into a threedimensional scaffolding under biological conditions. "We basically consider it to be a blank-slate scaffold," says Kosovsky, "meaning that customers can take it, make a gel, and add to it whatever they need to optimize the o environment for their cell type of interest." PuraMatrix has been successfully applied to the culture of a variety of primary and transformed cell lines, and has even shown some promise for tissue-engineering applications (Box 1).

There are no one-size-fits-all solutions, however. "We have a lot of papers showing that different kinds of extracellular matrix give different information to the nucleus," explains Bissell. Two groups at the Massachusetts Institute of Technology have recently described high-throughput, array-based screening approaches to explore this element of cell culture, examining how different cell types behave when subject to widely varying conditions. Robert Langer's group looked at fully artificial substrates, testing the effects of 576 different polymers on hESC growth and differentiation ${ }^{4}$, whereas Sangeeta Bhatia and colleagues used their platform to screen a variety of extracellular matrix formulations, incorporating different combinations of proteins like fibronectin, laminin and collagen ${ }^{5}$. Both approaches have proven informative for the analysis of suitable stem-cell culture conditions, and should be generalizable for optimizing growth and experimental strategies for other cell types as well.

\section{A moving experience}

For some research scenarios, a more dynamic system is better suited for the assembly and maintenance of three-dimensional, multicellular clusters, and Synthecon has parlayed its experiences in the development of microgravity simulators for the US National Aeronautics and Space Administration into an innovative series of culture instruments, the Rotary Cell Culture System (RCCS). "The basic system is a cylindrical culture vessel completely filled with culture medium, and it rotates horizontally," explains chief scientific officer Steve Navran. "The cells are put in suspension in the system and they don't grow on the surface unless you introduce some kind of carrier or scaffolding." Steady but gentle rotation keeps cells suspended in a lowshear environment that allows them to form aggregates of considerable size-as large as a centimeter in diameter, in some cases - and a gas-permeable silicone membrane provides bubble-free oxygenation. The RCCS has shown promise in a variety of tissue-engineering applications, including work with cartilage, blood vessels and the cultivation of pancreatic islet cells, but it is also suitable for basic cell biology research. A perfusion-based version is also available, in which medium is continually added to and removed from the culture, and this can be configured for more complex coculture experiments. "We've linked two of these chambers together, so you have two active cultures side by side that share the same nutrient loop," says CEO Bill

\section{BOX 1 GETTING ORGANIZED}

Many of the factors that now inform the way scientists develop cell-culture models also have a role in cell culture's more clinically oriented sibling, tissue engineering. Bhatia has been working in this area since the 1990s, and her experiences in cajoling cultured hepatocytes into maintaining an appropriate phenotype have helped her to zoom in on some primary determinants related to preserving cellular identity ex vivo. "We think it's a very short list," she says. "There are interactions with other cells, interactions with matrix, interactions with soluble factors, physical forces on different cell types, and what we sort of generically think of as 'the 3D context."

As with the culture applications described above, natural matrix proteins and polymeric analogs have been extensively tested for tissue modeling, organ reconstruction and even implantation into animals. More recently, synthetic hydrogels capable of in situ polymerization, including various polyethylene glycol-based materials, have become a promising option ${ }^{6}$; to further enhance appropriate cellular growth or differentiation, these hydrogels can also be laced with soluble growth factors or attractants. Last year, Bhatia and colleagues extended this approach by using dielectrophoretic forces to pattern cells three-dimensionally within an unpolymerized polyethylene glycol-based hydrogel solution ${ }^{7}$. Once the cells are appropriately positioned in space, the gel is rapidly photopolymerized, locking them in place and allowing more tightly controlled analysis of the effects of cell positioning and local density. Some of the bioreactor systems described above, such as Synthecon's RCCS and the various perfusion-based culture platforms, have also been used for the culture of tissue-like three-dimensional cell assemblies.
Most of the earliest successes to date have been with tissues that are defined by high matrix content, such as skin, cartilage and bone, whereas more complex tissues still lag behind. "It is probably not as hard to build mechanical structure, if that's your main output, as it is to re-establish metabolism," explains Bhatia. Scientists working with thicker tissues also face an additional challenge in the need to ensure adequate blood-vessel formation within the engineered organ. "I think that trying to build in new ways of vascularization is certainly one of the big issues," says Langer, "whether you do it by growth-factor engineering or controlled release, or whether you make nanoconduits by microfabrication." Langer and his colleagues in the field have tested a variety of solutions to this problem, including the use of complex cocultures of myoblasts, fibroblasts and endothelial cells on a polymer scaffold for the cultivation of transplantable, vascularized muscle grafts ${ }^{8}$, as well as the micropatterning and cultivation of capillaries on silicon wafers as a possible means for in vitro engineering of vascular networks ${ }^{9}$.

The challenges are different for each organ, and the solutions will obviously lie at the interface of cell biology and materials development. At the same time, however, there are also universal issues requiring general consideration, particularly from a therapeutic standpoint. "There's always an issue of biocompatibility," says Langer. "And from a cell standpoint, you'll have a choice where you might use the patient's own cells, and then you have the issue of supply, but if you take somebody else's cells, you have the issue of rejection... [and] those are some of the areas of active research that different groups are doing now." 
Adamson. "So you can have the first chamber secreting factors and other molecules that can be taken up in the second chamber, as in a helper-cell or feeder-cell format."

Provitro also offers a dual-chamber perfusion system, the Perfusion Chamber System $\mathrm{PCS}^{3 \mathrm{c}}$, which offers a useful platform for monitoring cell growth and migration in a coculture scenario. The design of this sys- tem allows users to work with a membrane or a well insert, which can be seeded and maintained in a multiwell plate before being transferred to the $\mathrm{PCS}^{3 \mathrm{c}}$ for further analysis. This platform is amenable to constant monitoring, explains managing director Manrico Paulitschke: "Since the chamber has windows, you can observe your culture positioned on a membrane between the two compartments with an inverted microscope." Provitro also offers two other perfusion systems. The Flow Chamber System FCS ${ }^{1 \mathrm{c}}$ is designed for investigating the effects of fluid shear forces on cultured cells, whereas the Tube Chamber System $\mathrm{TCS}^{2 \mathrm{c}}$ is designed for the cultivation of grafts from tissues such as blood vessels on a cylindrical support that provides the capacity for simultaneous perfusion from both an inner and outer circuit. These systems are typically connected to peristaltic pumps from Ismatec, which benefit from close computer control. "You can generate all kinds of regimes for pumping," says Paulitschke, "such as continuous with different shear rates, or different intervals and velocity, or you can even simulate pulsatile flow."

Active flow is also a key element of hollowfiber systems, in which medium is pumped through the interior of many porous membrane-based fibers. Hollow-fiber systems can successfully recreate physiological shear conditions in culture experiments, but are most commonly used as 'bioreactors' for bulk production of antibodies or recombinant proteins - culture processes with challenges of their own. "Hollow-fiber is the only way to grow cells in a post-confluent state for extended periods of time," says John Cadwell, president of FiberCell systems. "We've grown cells for over two years in continuous culture, and this is because the nutrients come from the bottom up." According to Cadwell, his company's products benefit from fibers that provide very high gross filtration rates, allowing ready exchange of nutrients and waste even when cells are densely layered on the outer surface of the fiber. These fibers are composed of either polysulfone or PS+, a proprietary membrane that is highly amenable to coating with a variety of molecules, including extracellular matrix proteins. PS+ has proven particularly advantageous for work with endothelial cells, which can be cultured on matrix inside the fibers to simulate vascular conditions. "If they grow in flask culture, after 120 hours they go through seven divisions," says Cadwell. "In our cartridge, they go through zero... they form a monolayer with tight junctions, and behave physiologically the way endothelial cells should."

Spectrum Laboratories will soon be relaunching their popular CellMax line of hollow-fiber systems with a host of new membrane formats with a wide range of molecular-weight cutoffs, as well as pumping systems capable of achieving greater flow rates-a key to effective aeration. One 


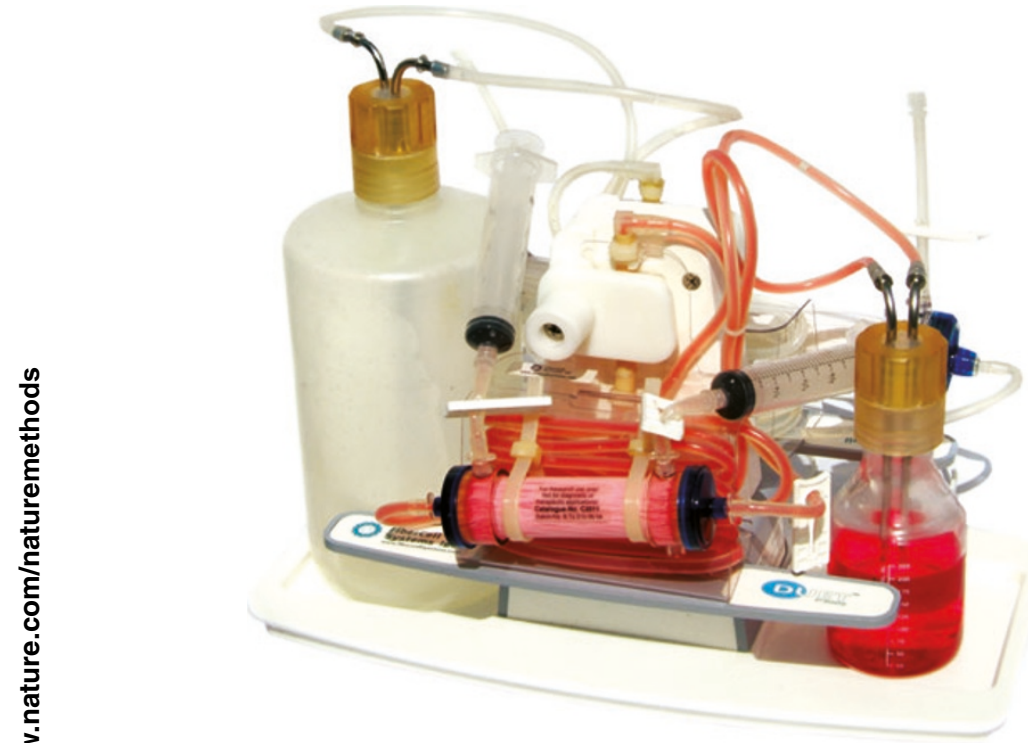

The Duet pump system from FiberCell Systems can support up to two hollow-fiber culture cartridges simultaneously. (Courtesy of FiberCell Systems.)

of the most widely requested membrane formats-regenerated cellulose-is also among the hardest to get, according to Bob Adamson, vice president of sales and marketing. "It's a pretty toxic process making it, and getting rid of the waste from this process is very costly," he says, "But we've acquired, both through ourselves and through others, several years' supply of it, and will continue to offer it." The closed nature of the hollow-fiber system yields advantages from a perspective of biosafety and preventing contamination, but again, cell yield is a key consideration. "If you inoculate $10^{6}$ cells into one of our smaller cartridges, you can get up to about $10^{10}$ or $10^{11}$ in a twelve-milliliter volume," says technical services manager Matthew Ré.

Hollow-fiber is not a universal solution, and issues include difficulty in scaling up and the impossibility of directly examining cells under cultivation. At the same time, however, for core facilities or companies interested in quick, medium-scale production runs, it provides a method for collecting high concentrations of protein that saves space and resources. "A start-up company doesn't have time to set up 200 roller bottles," says Cadwell, "they just need to produce the protein so they can test it and get an effect."

\section{Making a big production of it}

Traditionally, bulk culture processes have involved the use of numerous spinner flasks, standard tissue-culture 'T-flasks' or cylindrical roller bottles. Although these are still routinely used, they quickly pose problems as the desired protein quantity grows. "These really aren't scalable beyond a few liters because of issues of oxygen transfer and heat transfer and so forth," explains Vijay Singh, founder of Wave Biotech. As a result, users looking to work at a larger scale may benefit from more advanced 'stirred-tank' systems that can provide more elaborate oxygenation schemes and allow users to constantly monitor the health and well-being of the culture.

Sartorius offers a wide range of such instruments in their BIOSTAT family, with platforms capable of handling working volumes from 200-ml preparations all the way through to production-scale work. These include autoclavable benchtop units, such as the BIOSTAT B plus or the multivessel Q 12 unit, as well as units capable of in situ sterilization, like the benchtop BIOSTAT CT unit. The systems can be configured for a variety of culture scenarios, including the use of 'spin filters' for long-term perfusion culture.
Although the level of sophistication provided by these systems is generally geared toward research and development or production settings, Sartorius has recently launched a budget-friendly benchtop model, the BIOSTAT A plus, that could help make bioreactors more broadly accessible for entry-level users. "That starts at about $\$ 14,000$," says product manager Andre Grebe, "and it can be used at universities and start-up companies; it's a complete system, and even includes a laptop."

Microcarriers, small beads to which cells can bind in culture, are sometimes used in bioreactors as a means to culture adherent cells at considerably elevated densities. A variety of such products, based either on synthetic polymers or natural scaffolds like gelatin, dextran or cellulose, are available from companies like GE Healthcare, Percell and Nunc. Microcarriers can be difficult to work with and are prohibitively expensive for long-term use, but they remain popular for certain applications, such as vaccine production, and provide a clear boost in culture productivity. New Brunswick Scientific has expanded on this technology with the development of their FibraCel polymeric disks, which provide a porous three-dimensional support for the production of secreted proteins from a variety of anchorage-dependent or suspension cell lines. The company claims that the large surface area provided by the three-layered structure of these disks provides up to ten times the productivity of standard microcarriers, as well as increased protection against shear.

FibraCel disks are routinely used for protein production applications in New Brunswick's range of bioreactor systems. The newest of these, the CelliGen 310, is a benchtop system that can independently control four culture vessels simultaneously. New Brunswick offers a range of impellers to provide appropriate agitation for various standard culture scenarios, including a patented

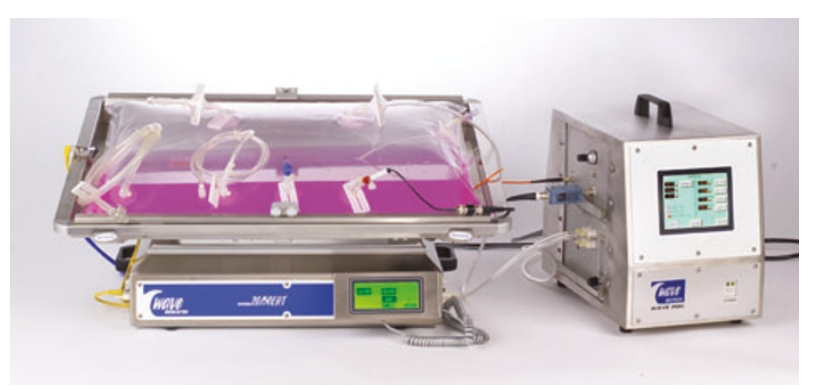

The Wave Bioreactor 20/50 EHT offers one solution for mid-range production for users interested in working in a disposable format. (Courtesy of Wave Biotech.) 
Cell-Lift impeller for performing perfusion culture with microcarriers or cells in suspension. The CelliGen 310 comes equipped with a touch-screen interface that can be used to control detailed cell culture recipes and manage input from a variety of sources. "You can connect things like cell-mass probes, turbidity probes or a $\mathrm{CO}_{2}$ probe," says product manager Rajul Vyas. "In addition, we provide the option of integrating eight digital systems, such as scales, directly into the bioreactor's control loops. In all, we've built in the capability for controlling up to 32 parameters per vessel."

\section{Productivity at your disposal}

There is also a need for solutions that straddle the line between traditional culture vessels and high-end, automated bioreactors. "Many people know they need to move to some type of bioreactor, but they don't want to move to a great big piece of plant with all of its complexities and costs," explains Cellexus Biosystems CEO Kevin Auton, "because often, the production is very transient."

Both Sartorius and New Brunswick have developed disposable culture solutions for production that complement their higherend systems. New Brunswick offers their 500ml FibraStage bottles, which feature a unique 'bellows' design. When these are placed into the FibraStage culture device, the unit automatically compresses and releases the bellows, gently pushing the medium back and forth through a permeable central culture cham-

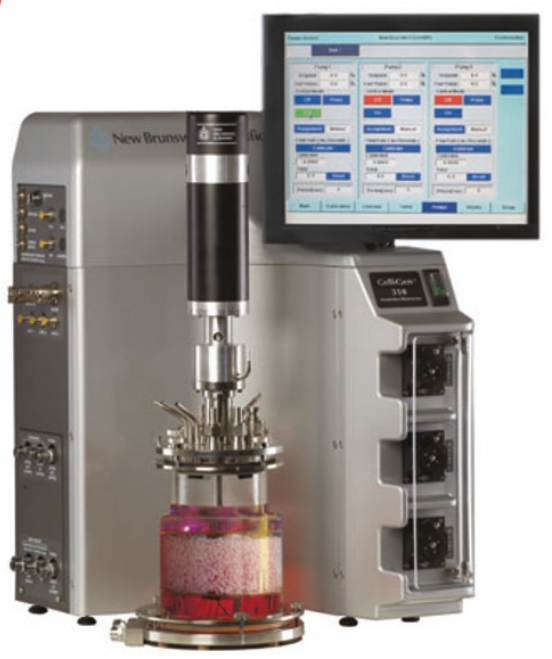

New Brunswick Scientific's CelliGen 310, fitted with a packed-bed basket that uses the company's FibraCel disks for increased culture density and productivity. (Courtesy of New Brunswick Scientific.) ber, which is preloaded with FibraCel disks. This process provides both oxygen and nutrients to cultured cells, and the whole setup can readily be placed in a standard incubator. "Customers don't need to use high-volume cell-culture techniques," says Vyas, "and we usually say that one FibraStage bottle can replace 20-40 roller bottles, depending on your cell line."

In the months ahead, Sartorius also will be launching two disposable bioreactor product lines. These include a disposable, actively aerated spinner flask for general protein production, as well as a series of shaker vessels designed for small-scale experimentation or cell-culture process development and optimization. This latter product, known as the CultiFlask line, is designed for use in a specialized incubation shaker that can maintain appropriate environmental conditions for mammalian cell work. "This is not something that aims to replace big systems, but rather complements them," explains product manager Joachim Luecke. "Some companies are a little hesitant to spend money on large stainless steel reactors without knowing whether their product idea will come to anything."

Newcomer Cellexus is targeting academics, small companies and other users on a budget with the CellMaker Lite, a bag-based culture system with an innovative aeration scheme that provides a cost-effective bioreactor setup. The bag is shaped like an inverted $\mathrm{L}$ and is aerated at the bottom in a configuration that provides proper oxygenation without mechanical agitation. "It's arranged such that the airstream is in a long ribbon that hits a deflector, and this gives you good mixing into all parts of the bioreactor," explains Auton. "And it has a very large surface area compared with the volume..., which means you also achieve gas exchange from the surface." The bag is maintained within a temperature-controlled enclosure, and is pressurized to promote aeration of the culture medium while considerably reducing foaming, a leading cause of cell death. Current CellMaker models are designed for bare-essentials production at 1-8-1 or 10-50-1 culture volumes, but Cellexus has planned more heavily automated, current good manufacturing processes (cGMP)compliant system for the near future.

Culture bags are also the core of Wave Biotech's line of bioreactors, which gently rock media and cells to provide mixing and aeration for volumes ranging from $100 \mathrm{ml} \mathrm{up}$ to 5001 . "As the wave travels, it entrains air in a dissolved fashion, so you don't have the bubble issues you have with a sparge system," explains Singh, "and since there's no propeller, there's no local shear gradient." The bags themselves are simple, but take advantage of advanced mechanical design in the rockers that minimizes the potential for cell damage resulting from the movement of the media, and the bags also incorporate valves for the management of culture gassing conditions or the attachment of sensors and monitoring equipment. More recently, Wave introduced optional floating perfusion filters, which make longer-term culture a possibility. According to Singh, this configuration has proven particularly popular for the production of gene-therapy products. "We typically have people getting between fifty and seventy million cells per milliliter," he says, "and it's a two- to three-week operation."

According to Florian Wurm, a bioreactor specialist at the Swiss Federal Institute of Technology at Lausanne, such systems have enabled even users with little experience in culture process development to achieve exceptional protein production-but he adds that technology is only part of the reason for this. "It's actually the cells...; we now have somewhere between 10 and 50 times more cells in a reactor today than we had twenty years ago," says Wurm. "Our processes have obtained a degree of sophistication in terms of understanding what cells need that is unparalleled." Singh agrees, adding that this outcome has proven beneficial for both manufacturers confronting scale-up issues and users craving efficiency. "The volume of bioreactors is shrinking, and the yield in cell cultures is rising," he says. "Ten years ago, if you went to a conference and somebody told you they had one gram per liter of antibody, you'd clap; now if you get less than six, they'll say, 'Come on!'”

1. Kleinman, H.K. et al. Biochemistry 25, 312-318 (1986).

2. Petersen, 0.W. et al. Proc. Natl. Acad. Sci. USA 89, 9064-9068 (1992).

3. Ludwig, T.E. et al. Nat. Biotechnol. 24, 185-187 (2006).

4. Anderson, D.G. et al. Nat. Biotechnol. 22, 863866 (2004).

5. Flaim, C.J. et al. Nat. Methods 2, 119-125 (2005)

6. Lutolf, M.A. \& Hubbell, J.A. Nat. Biotechnol. 23, 47-55 (2005).

7. Albrecht, D.R. et al. Nat. Methods 3, 369-375 (2006).

8. Levenberg, S. et al. Nat. Biotechnol. 23, 879-884 (2005).

9. Fidkowski, C. et al. Tissue Eng. 11, 302-309 (2005).

Michael Eisenstein is technology editor for Nature and Nature Methods. 


\section{SUPPLIERS GUIDE: COMPANIES OFFERING CELL-CULTURE EQUIPMENT, PRODUCTS OR SERVICES}

Company
Amerex Instruments
American Type Culture Collection
Applikon Biotechnology
Argos Technologies
AthenaES

B-Bridge International

BD Biosciences

BINDER

Bio Express

Bionique Testing Laboratories, Inc.

Cambrex

Cascade Biologics

Celartia

Cell Culture Solutions

Cell Culture Technologies

Cell Essentials

Cellexus Biosystems

을 Cell Trends

Cellutron

Corning Life Sciences

European Collection of Cell Cultures

FiberCell Systems

Fisher Scientific

Flexcell International

Fluorometrix

Genomic Solutions

Web address

http://www.amerexinst.com

http://www.atcc.org

http://www.applikonbio.com

http://www.argos-tech.com

http://www.athenaes.com

http://www.b-bridge.com

http://www.bdbiosciences.com

http://www.binder-world.com/

international/en

http://www.bio-express.com

http://www.bionique.com

http://www.cambrex.com

http://www.cascadebio.com

http://www.celartia.com

http://www.cellculturesolutions.com

http://www.cellculture.com

http://www.cell-essentials.com

http://www.cellexusbiosystems.com

http://www.celltrends.com

http://www.cellutron.com

http://www.corning.com/lifesciences

http://www.ecacc.org.uk

http://www.fibercellsystems.com

http://www.fishersci.com

http://www.flexcellint.com

http://www.fluorometrix.com

http://www.genomicsolutions.com

German Collection of Microorganisms http://www.dsmz.de

and Cell Cultures

(2) Greiner Bio One

HyClone BPC

O0 INCELL

Integra Biosciences

Invitrogen

InvivoGen

Major Science http://www.gbo.com

http://www.hyclone.com

http://www.incell.com

http://www.integra-biosciences.com

http://www.invitrogen.com

http://www.invivogen.com

http://www.major-science.com
Company

Memmert

MIDSCI

Millipore

Miltenyi Biotec

Minucells and Minutissue Vertriebs

$\mathrm{GmbH}$

Mycoplasma Experience Ltd.

National Cell Culture Center

New Brunswick Scientific

Nexcelom Biosciences

NuAire

Nunc Brand Products

Ortec International

Osmotek

Paragon Bioservices

PromoCell

Provitro

\section{R\&D Systems}

Roche Applied Science

RS Biotech

Sanyo Biomedical

Sartorius

Sigma-Aldrich

Spectrum Laboratories

StemCell Technologies

Synthecon

Thermo Electron

3DM Synthetic Nanoscaffolds, Inc.

Trevigen

Valley Biomedical

VWR

Wave Biotech

Wheaton Science

WiCell Research Institute/National

Stem Cell Bank
Web address

http://www.memmert.com

http://www.midsci.com

http://www.millipore.com

http://www.miltenyibiotec.com

http://www.minucells.de

http://www.mycoplasma-exp.com

http://www.nccc.com

http://www.nbsc.com

http://www.cellometer.com

http://www.nuaire.com

http://www.nuncbrand.com

http://www.ortecinternational.com

http://www.osmotek.com

http://www.paragonbioservices.com

http://www.promocell.com

http://www.provitro.de

http://www.rndsystems.com

http://www.roche-applied-science.com http://www.rsbiotech.com

http://www.sanyo.com/biomedical

http://www.sartorius.com

http://www.sigmaaldrich.com

http://www.spectrapor.com

http://www.stemcell.com

http://www.synthecon.com

http://www.thermo.com

http://www.puramatrix.com

http://www.trevigen.com

http://www.valleybiomedical.com

http://www.vwr.com

http://www.wavebiotech.com

http://www.wheatonsci.com

http://www.wicell.org 